\title{
Modeling the damming effect on hydrological alteration and prediction of discharge in Padma River by proposing PSO based novel hybrid machine learning algorithm
}

\author{
Abu Reza Md. Towfiqul Islam ${ }^{1}$, Swapan Talukdar², Shumona Akhter ${ }^{1}$, Kutub Uddin \\ Eibek ${ }^{1}$, Md. Mostafizur Rahman ${ }^{3}$, Ashraf Dewan ${ }^{4}$, Quoc Pham ${ }^{5}$, Nguyen Thi Thuy Linh ${ }^{6}$, \\ Swades Pal ${ }^{2}$, Thi Ngoc Canh DOAN ${ }^{7}$, Duong Tran $\mathrm{Anh}^{8}$, and Sobhy M. Ibrahim ${ }^{9}$ \\ ${ }^{1}$ Begum Rokeya University \\ ${ }^{2}$ University of Gour Banga \\ ${ }^{3}$ Jahangirnagar University \\ ${ }^{4}$ Curtin University \\ ${ }^{5}$ National Cheng-Kung University \\ ${ }^{6}$ Duy Tan University \\ ${ }^{7}$ The University of Danang University of Economics \\ ${ }^{8}$ Ton Duc Thang University \\ ${ }^{9}$ King Saud University
}

September 24, 2020

\begin{abstract}
This paper quantified the hydrological alteration of the Padma River basin caused by the construction of Ferakka Barrage (FB) using innovative trend analysis (ITA), range of variability approach (RVA), and continuous wavelet analysis (CWA). We also predict flow regime by proposing particle swarm optimization (PSO) based novel hybrid machine learning algorithms. Results of the ITA showed the negative trend of the average discharge in the dry season (January-May), while the RVA analysis indicated that average discharge was lower than environmental flows. The CWA demonstrated a substantial effect of the FB on the periodicity of the streamflow regime. Results showed that PSO-Reduced Error Pruning Tree (REPTree), PSO-random forest (RF), and PSO-M5P were the optimal fit for average, maximum, and minimum discharge prediction $(\mathrm{RMSE}=0.14,0.3,0.18)$ respectively.
\end{abstract}

Modeling the damming effect on hydrological alteration and prediction of discharge in Padma River by proposing PSO based novel hybrid machine learning algorithm

Abu Reza Md. Towfiqul Islam ${ }^{1}$, Swapan Talukdar ${ }^{2}$, Shumona Akhter ${ }^{1}$, Kutub Uddin Eibek ${ }^{1}$, Md. Mostafizur Rahman $^{3}$, Ashraf Dewan ${ }^{4}$, Quoc Bao Pham ${ }^{5,6}$, Nguyen Thi Thuy Linh ${ }^{7,8^{*}}$, Swades $\mathrm{Pal}^{9}$, Thi Ngoc Canh DOAN $^{10}$, Duong Tran Anh ${ }^{11}$, Sobhy M. Ibrahim ${ }^{12,13}$

This study quantified the hydrological alteration of the Padma River basin caused by the construction of Ferakka Barrage using different statistical models. The negative trend of the average discharge in the dry season was detected, while the average discharge was lower than environmental flows. PSO based ensemble machine learning models were developed. PSO-Reduced Error Pruning Tree (REPTree), PSO-random forest $(\mathrm{RF})$, and PSO-M5P were the optimal fit for average, maximum, and minimum discharge prediction (RMSE $=0.14,0.3,0.18)$ respectively. 


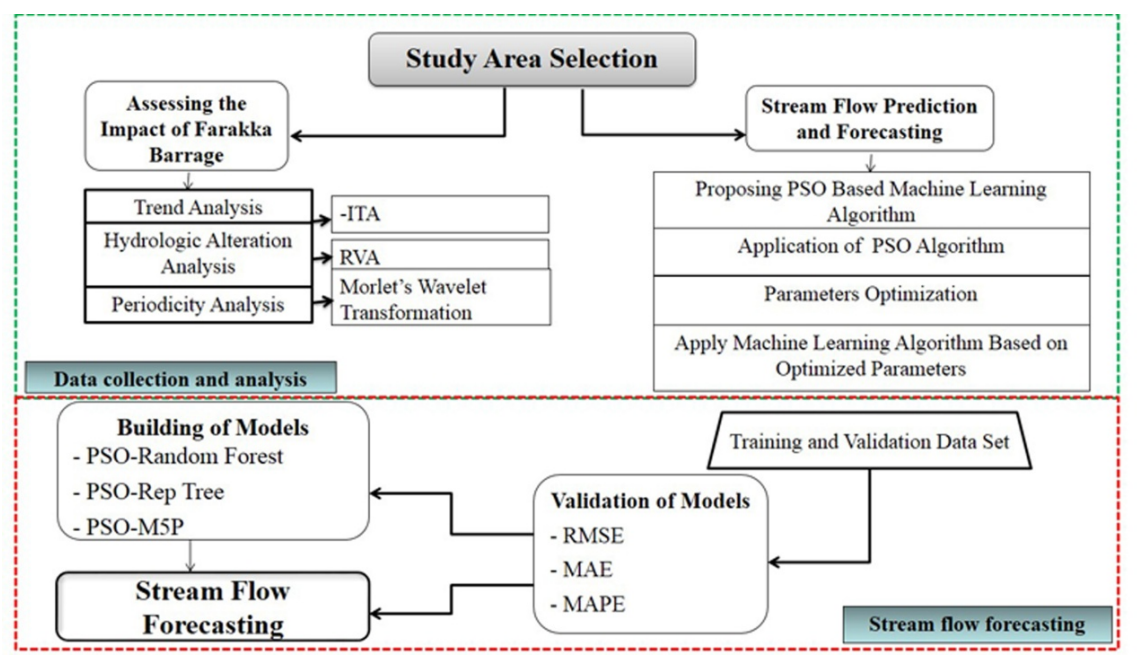

\section{Hosted file}

Hydrology Manuscript.pdf available at https://authorea.com/users/353697/articles/482693modeling-the-damming-effect-on-hydrological-alteration-and-prediction-of-discharge-inpadma-river-by-proposing-pso-based-novel-hybrid-machine-learning-algorithm 